\title{
A Typical Angiosarcoma of the Posterior Mediastinum: One Case Report and Review of the Literature
}

\section{Qingqiang Zhu*, Jingtao Wu, Wenrong Zhu, Wenxin Chen and Shouan Wang}

Department of Medical Imaging, Subei People's Hospital, Medical School of Yangzhou University, Yangzhou, China

\begin{abstract}
Angiosarcoma of the posterior mediastinum is a rare neoplasm of soft tissues and its location in the mediastinum is extremely uncommon. According to the papers of other cases reported on angiosarcoma in mediastinum, invasive biological behavior exist, such as ill-defined margin, heterogeneous density and local involvement of lung and ribs or vertebra. This case showed a well-defined, homogeneous mass. So, confusion is possible with other benign tumor in mediastinum.
\end{abstract}

Keywords: Mediastinal tumor; Angiosarcoma; Computerized Tomography (CT); Pathology

\section{Introduction}

Angiosarcomas are most often encountered as sporadic cutaneous lesions, typically in the scalp or face of an elderly patient. However, angiosarcomas can occur in virtually any anatomic site, including the deep soft tissue, breast, visceral organs, and bone. Primary

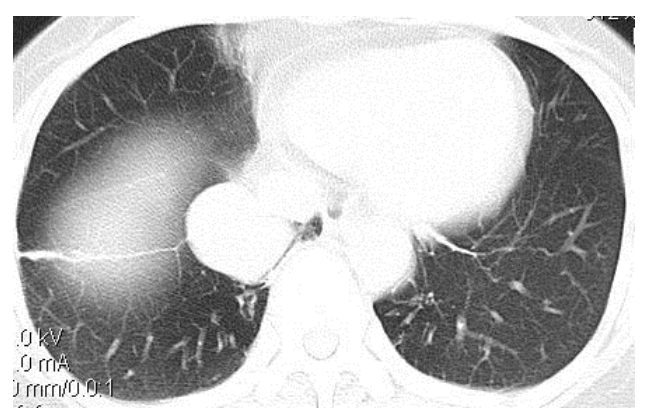

Figure 1: Unenhanced computerized tomography (CT) showed a welldefined $3.8 \mathrm{~cm}^{*} 3.0 \mathrm{~cm}^{*} 3.2 \mathrm{~cm}$ homogeneous hyper dense mass in the right posterior mediastinum, where was close to the spine and measured about $54 \mathrm{HU}($ arrow). Unenhanced computerized tomography (CT) showed a welldefined $3.8 \mathrm{~cm}^{*} 3.0 \mathrm{~cm} 3.2 \mathrm{~cm}$ homogeneous hyper dense mass in the right posterior mediastinum, where was close to the spine and measured about $54 \mathrm{HU}($ arrow)..

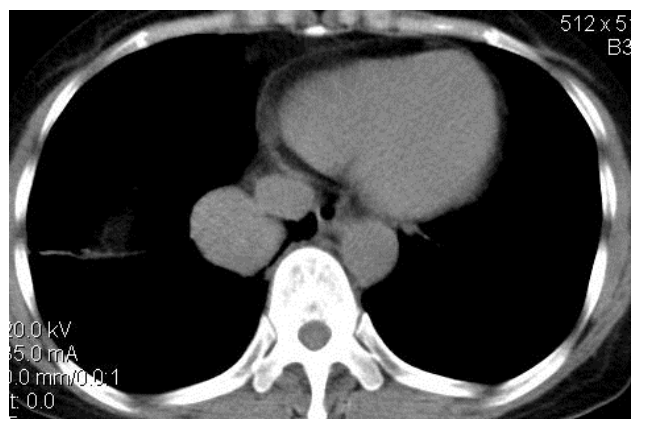

Figure 1: Unenhanced computerized tomography (CT) showed a welldefined $3.8 \mathrm{~cm}^{*} 3.0 \mathrm{~cm}^{*} 3.2 \mathrm{~cm}$ homogeneous hyper dense mass in the right posterior mediastinum, where was close to the spine and measured about $54 \mathrm{HU}$ (arrow). Unenhanced computerized tomography (CT) showed a welldefined $3.8 \mathrm{~cm}^{*} 3.0 \mathrm{~cm} * 3.2 \mathrm{~cm}$ homogeneous hyper dense mass in the right posterior mediastinum, where was close to the spine and measured about $54 \mathrm{HU}($ arrow).. angiosarcomas of the posterior mediastinum are extremely rare. According to the papers of other cases reported on angiosarcoma in mediastinum, invasive biological behavior exist, such as ill-defined margin, heterogeneous density and local involvement of lung and ribs or vertebra. This case showed a well-defined, homogeneous mass without metastasis. We herein report an atypical case of the posterior mediastinum and present the CT imaging, clinicopathologic and immunohistochemical features of this tumor. In addition, the differential diagnoses are discussed.

\section{Case Reports}

A female, 56 years old. For the medical examination, the right posterior mediastinum mass was found more than one month and was hospitalized on Oct 23, 2009. On admission, the patient appeared in good general condition. There was no history of cigarette smoking and no family history of malignancy. There was also no history of occupational exposure to carcinogens. More than one month ago, the patient began to appear blurry vision, without improving after having a break. In the local hospital, she was given a symptomatic treatment. During the course of disease, the patient had no nausea, vomiting, no fever, cough, night sweats, no hoarseness, dyspnea, no weight loss and other symptoms. Physical examination was normal. The right posterior mediastinum tumor had been resected on Oct 28, 2009 and postoperative radiotherapy was taken. The patient followed up so far and recovered well.

Unenhanced Computerized Tomography (CT) showed a welldefined $3.8 \mathrm{~cm}^{\star} 3.2 \mathrm{~cm} * 3.0 \mathrm{~cm}$ homogeneous hyper dense mass in the right posterior mediastinum, where was close to the spine and measured about $54 \mathrm{HU}$ (Figures 1 and 2). On enhanced CT, the tumor showed mild homogeneous enhancement on arterial phase, which was measured about $66 \mathrm{HU}$ (Figure 3 ). The vermicelli-shaped blood vessels were seen in the central mass (Figure 3, arrow). The enhancement of the tumor was stratified on venous phase (Figure 4). Marked enhancement in the marginal tumor, which measured about $87 \mathrm{HU}$ (Figure 4 black

*Corresponding author: Qingqiang Zhu, Department of Medical Imaging, Sube People's Hospital, Medical School of Yangzhou University, Yangzhou, China, Tel: 86-514-87373625; Fax: 86-514-87373625; E-mail: zhuqingqiang1983@163.com

Received February 18, 2014; Accepted July 18, 2014; Published July 21, 2014

Citation: Zhu Q, Wu J, Zhu W, Chen W, Wang S (2014) A Typical Angiosarcoma of the Posterior Mediastinum: One Case Report and Review of the Literature. J Clin Case Rep 4: 387. doi:10.4172/2165-7920.1000387

Copyright: @ 2014 Zhu Q, et al. This is an open-access article distributed under the terms of the Creative Commons Attribution License, which permits unrestricted use, distribution, and reproduction in any medium, provided the original author and source are credited. 
Citation: Zhu Q, Wu J, Zhu W, Chen W, Wang S (2014) A Typical Angiosarcoma of the Posterior Mediastinum: One Case Report and Review of the Literature. J Clin Case Rep 4: 387. doi:10.4172/2165-7920.1000387

Page 2 of 3

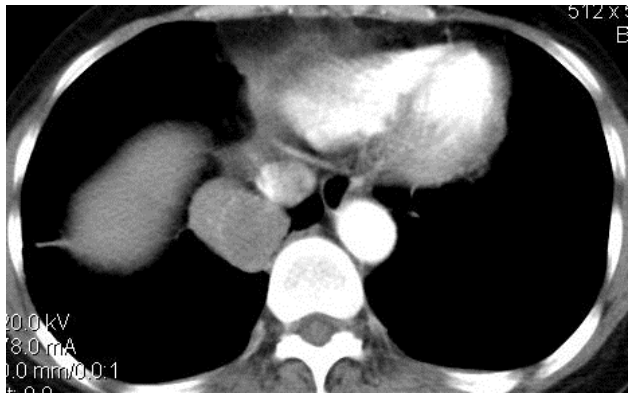

Figure 3: On enhanced CT, the tumor showed mildly homogeneous enhancement on arterial phase, which was measured about $66 \mathrm{HU}$. The vermicelli-shaped blood vessels were seen in the central mass (arrow).

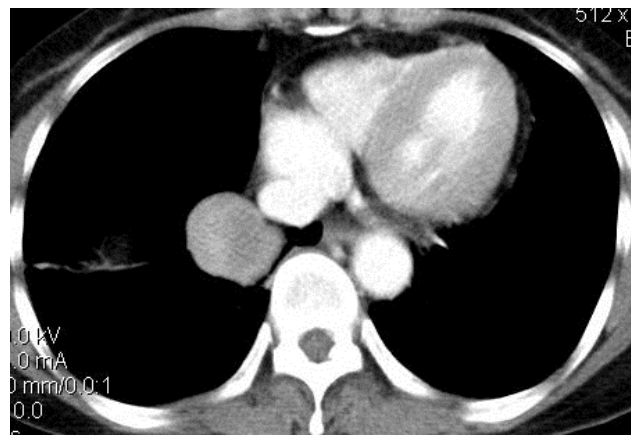

Figure 4: The enhancement of the tumor was stratified on venous phase. Marked enhancement in the marginal tumor, which measured about $87 \mathrm{HU}$ (black arrow). While mild enhancement in central tumor, which measured about $58 \mathrm{HU}$ (white arrow).

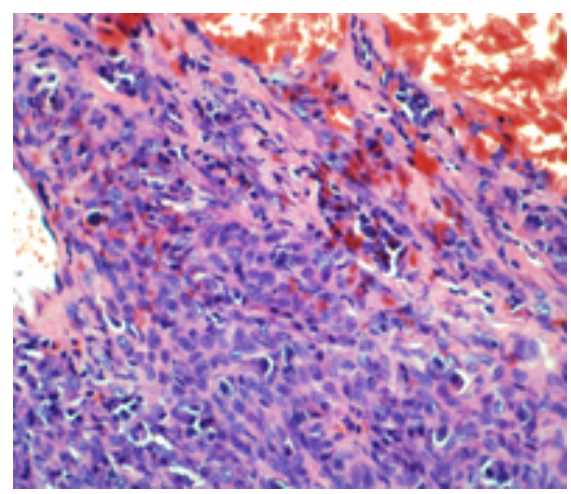

Figure 5: Microscopic findings of the tumor showed vascular proliferation, expansion, congestion and hemorrhage. The cells showed cords, nests pattern growth with mild atypical nucleus. Slit-shaped small blood vessel and blood capillary were seen in intercellular (HE×400).

arrow). While mild enhancement in central tumor, which measured about 58 HU (Figure 4 white arrow).

Microscopic findings of the tumor showed vascular proliferation, expansion, congestion and hemorrhage in high-grade. The tumor cells showed cords, nests pattern growth with mild atypical nucleus. Slitshaped small blood vessel and blood capillary were seen in intercellular (Figure 5). Immunochemistry staining demonstrated CD34 ${ }^{++}, \mathrm{CD}^{++}$, FLI- ${ }^{+++}$(Figure 6), $\mathrm{PCNA}^{++}$(Figure 7), Vimentin ${ }^{++}$, and were negative for VIII factor protein.

\section{Discussion}

Angiosarcoma is an uncommon tumor representing less than $1 \%$ of all sarcomas and the most common clinical presentation is as a cutaneous tumor in the scalp and facial regions of elderly men [1]. Primary angiosarcomas arising in the mediastinum are very rare. Median age at presentation is 52 years and the prognosis is generally poor with a median survival of 42 months and a 5 -year overall survival of $43 \%$ [2]. Chest pain was the main presenting symptom and, from the limited data available, survival ranged from 2 to 36 months from time of diagnosis [3-4]. We have studied a further one case of angiosarcoma arising in the posterior mediastinum.

Mediastinal angiosarcomas were mainly located in the anterior mediastinum, and they were divided into epithelioid hemangioendothelioma type and spindle cell hemangioendothelioma type [5]. Because of the high degree of malignancy, the surrounding tissues adjacent to the lesion are prone to have metastasis at the time of presentation. Common metastatic sites include the regional lymph nodes, lungs and bones. The cause of the disease is not clear at present. The long term chronic lymphedema, ionizing radiation, chemical exposure, trauma, chronic infection and other factors may be relative to the disease [6].

Angiosarcoma in mediastinum, invasive biological behavior exist, such as ill-defined margin, heterogeneous density and local involvement of lung and ribs or vertebra. Atypical angiosarcoma is more a single mass, round or nodular, and can diffuse infiltration into adjacent

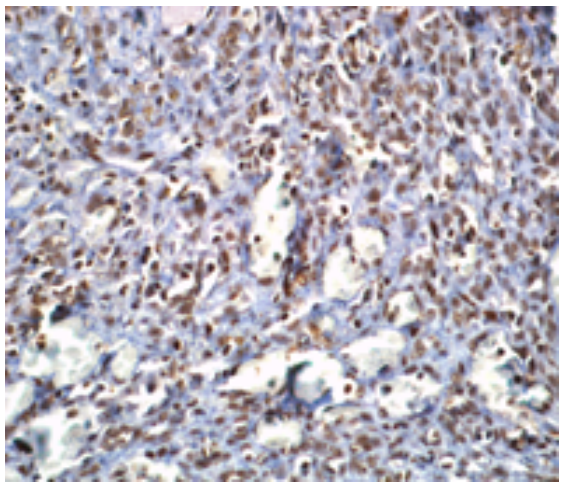

Figure 6: Immunochemistry staining demonstrated $\mathrm{FLI}-1^{+++}$, SP method

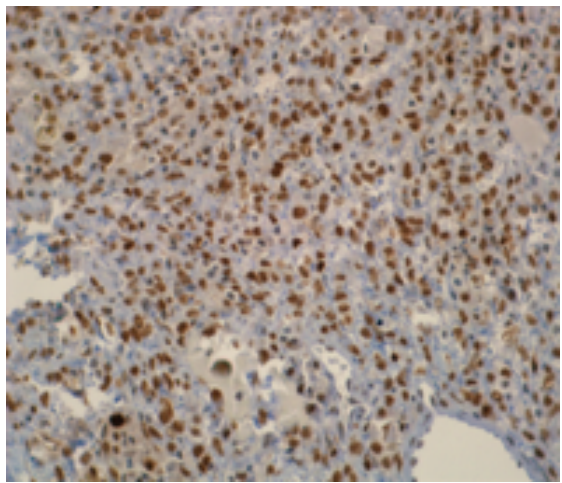

Figure 7: Immunochemistry staining demonstrated $\mathrm{PCNA}^{++}, \mathrm{SP}$ method. 
tissues. Clinically, the patients all presented with the typical symptoms of mediastinal masses, namely, chest pain, dyspnea, and cough.

An oval tumor exhibiting vermicelli-shaped blood vessels, stratified enhancement, may be considered as specific CT imaging features of atypical angiosarcoma of the mediastinum. The final diagnosis depends on pathological examination. CD31, CD34 and other vascular markers can help to confirm the diagnosis of the disease [7].

Currently, a single treatment is ineffective. The combination of operation, radiotherapy or chemotherapy was key factors to improve the outcomes. Some scholars believed that the chemotherapy for this disease was invalid. However, some scholars think it is still a necessary palliative therapeutic tool [8]. In recent years, some scholars believe that Avastin is one of effective drugs in treatment of angiosarcoma [9]. Judging from our case treated, the combination of operation and radiotherapy may have certain advantages in improving the quality of life.

The most important differential diagnosis of the angiosarcoma is epithelioid hemangioendothelioma [10]. Epithelioid hemangioendothelioma is a vascular neoplasm of low to intermediate malignant potential that follows a nonaggressive clinical course. This tumor has been described in the soft tissue as well as in various other organ systems including bone, liver, and lung. The tumor may run a less aggressive clinical course, whereas angiosarcoma has a much higher potential for progression and metastasis. Angiosarcomas also need to be differentiated from carcinoma. Carcinomas, whether primary or metastatic, lack a vasoformative component and express an epithelial immunohistochemical phenotype; they show no reactivity for vascular markers [11].

Information regarding the clinical behavior of angiosarcoma of the mediastinum is limited due to there rare incidence [12]. Among our case of angiosarcoma, the patient is currently still alive without recurrence or metastasis. Although the clinical course of this patient is rather indolent, routine follow-up is still mandatory. The correct distinction of angiosarcomas from other tumors can lead to better understanding of their clinicopathologic differences, which should aid in developing individualized management plans.

In conclusion, angiosarcoma of the posterior mediastinum is a rare tumor with special pathologic features. Precise diagnosis based on CT imaging findings alone remains difficult. However, we clearly demonstrated imaging features that may help to distinguish the tumor.

\section{References}

1. Gatcombe HG, Olson TA, Esiashvili N (2010) Metastatic primary angiosarcoma of the breast in a pediatric patient with a complete response to systemic chemotherapy and definitive radiation therapy: case report and review of the literature. Pediatr Hematol Oncol 32: 192-194.

2. Ferrari A, Casanova M, Bisogno G, Cecchetto G, Meazza C, et al. (2002) Malignant vascular tumors in children and adolescents: a report from the Italian and German Soft Tissue Sarcoma Cooperative Group. Med Pediatr Oncol 39 109-114.

3. Ayadi L, Khabir A (2010) Pediatric angiosarcoma of soft tissue: a rare clinicopathologic entity. Arch Pathol Lab Med 134: 481-485.

4. Deyrup AT, Miettinen M, North PE, Khoury JD, Tighiouart M, et al. (2009) Angiosarcomas arising in the viscera and soft tissue of children and young adults: a clinicopathologic study of 15 cases. Am J Surg Pathol 33: 264-269.

5. Ikeda K, Maehara M, Ohmura N, Kurokawa H, Koda K, et al. (2006) Spontaneous rupture of a necrotic hepatic angiosarcoma: findings on dual-phase computed tomography and angiography. Radiat Med 24: 369-372.

6. Lejeune FJ, Pujol N, Liénard D, Mosimann F, Raffoul W, et al. (2000) Limb salvage by neoadjuvant isolated perfusion with TNFalpha and melphalan for non-resectable soft tissue sarcoma of the extremities. Eur J Surg Oncol 26 669-678.

7. Suzuki Y, Yoshida YK, Shirane R, Yoshimoto T, Watanabe M, et al. (2000) Congenital primary cerebral angiosarcoma. Case report. J Neurosurg 92: 466468.

8. Naka N, Ohsawa M, Tomita Y, Kanno H, Uchida A, et al. (1996) Prognostic factors in angiosarcoma: a multivariate analysis of 55 cases. J Surg Oncol 61 : 170-176.

9. Morgan MB, Swann M, Somach S, Eng W, Smoller B (2004) Cutaneous angiosarcoma: a case series with prognostic correlation. J Am Acad Dermato 50: 867-874.

10. Ferrari A, Miceli R, Meazza C, Zaffignani E, Gronchi A, et al. (2009) Soft tissue sarcomas of childhood and adolescence: the prognostic role of tumor size in relation to patient body size. J Clin Oncol 27: 371-376.

11. Chen TJ, Chiou CC, Chen CH, Kuo TT, Hong HS (2008) Metastasis of mediastinal epithelioid angiosarcoma to the finger. Am J Clin Dermatol 9: 181183.

12. Fayette J, Martin E, Piperno-Neumann S, Le Cesne A, Robert C, et al. (2007) Angiosarcomas, a heterogeneous group of sarcomas with specific behavio depending on primary site: a retrospective study of 161 cases. Ann Oncol 18 : 2030-2036. 